\title{
SYSTEM CONTROL IN FAULT AND NORMAL CONDITIONS \\ CASE STUDY - QUANSER SRV-02
}

\author{
RESCEANU Ionut Cristian \\ assistant professor,Faculty of Automation Computers and Electronics/Department of Mechatronics and \\ Robotics, University of Craiova, Craiova, Romania, resceanu@robotics.ucv.ro \\ RESCEANU Cristina Floriana \\ assistant professor, Faculty of Automation Computers and Electronics/Department of Mechatronics and \\ Robotics, University of Craiova, Craiova, Romania, cristina@robotics.ucv.ro
}

\begin{abstract}
A fault tolerant control method is proposed for Quanser SRV-02 System in order to maintain the required performance in the presence of sensor failures. The proposed approach integrates control law and a sensor fault tolerance schema. Theoretical analysis and simulation results have confirmed the effectiveness of the proposed method.
\end{abstract}

Key words: Fault tolerant systems, Control systems

\section{Introduction}

The Quanser SRV-02 series is ideal for introducing fundamental control principles and theories. The plant contains a MicroMo Coreless DC Motor encapsulated in a strong aluminum frame. This model is a high efficiency low inductance motor resulting in a much faster response than a conventional DC motor. The motor is equipped with a gearbox that drives external gears. The motor connection is a 4-pin DIN connector configured to be driven by a Quanser Universal Power Module.

Attached to the system is the encoder that measures $1024 \times 4$ impulses in a complete rotation. This means that 360 degrees are equivalent to $1024 \times 4=4096$ impulses. The resolution of the encoder counter is $360 / 4096=0.0878906250$ degrees. The model used during the experiments is a US Digital Optical Encoder kit. [7]

\section{Protection methods for faulty transducers}

Transducer function validation checks the difference between two signals provided by two transducers placed on the same size controlled signals which in principle should be equal as in [1],[2]. If the difference exceeds a threshold value determined a priori, an alarm signal is generated after a time. Where there are several sensors using the principle of majority voting is possible automatically be removed from the defective transducer control structure (its signal is ignored).

A possible technical solution is the simultaneous use of two transducers. If $p$ for sensor failure probability, then the simultaneous failure of both, considered as independent events, is likely $p$ two obviously lower than $p$.

It considers the structure in Figure 3. Significance of building blocks is as follows:

- $\quad R$ - controller;

- $E E$ - actuator;

- Process - Automated installation technology;

- $\quad T_{1}$ and $T_{2}$ - identical transducers $Y$ same size;

- $\mathrm{DB}$ - decision block

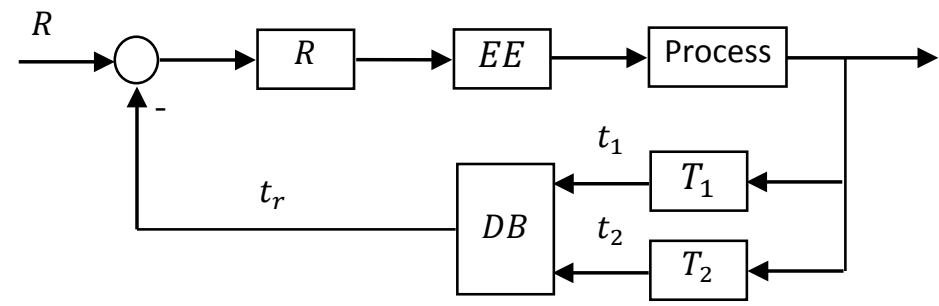

Figure 1: Control Structure 
The control scheme used for the decision block was proposed by Iancu and Vînătoru [3], [4] and is illustrated in the following figure:

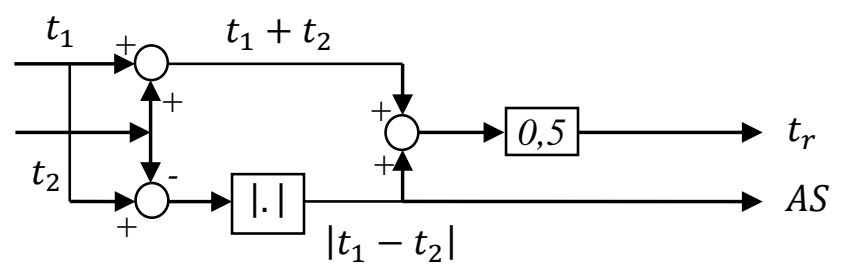

Figure 2: Control Structure

This structure helps maintain a continuous signal path of reaction, even when information provided by the transducer is no longer available. When both sensors are working normally and their signals are roughly equal in size $t_{\mathrm{r}}$ reaction is given by the following:

$t_{r}=0,5\left(t_{1}+t_{2}\right)$

each sensor with a contribution of $50 \%$. When a sensor contact is interrupted (e.g. $t_{2}=0$ ) signal $t_{\mathrm{r}}$ becomes:

$t_{r}=0,5\left(2 t_{1}\right)=t_{1}$

Similarly, for the case when a $=0, t$ reaction becomes equal to $t_{2}$. Meanwhile, AS signal can be used as an alarm signal under the convention:

- AS = 0 , function properly;

- AS $=1$, defective functioning.

This scheme will select the output size of the entry that has the highest value.

Another structure is shown in 3 and 4 is equivalent to having plus size model providing $t_{m}$ process used to detect large deviations of the transducer.

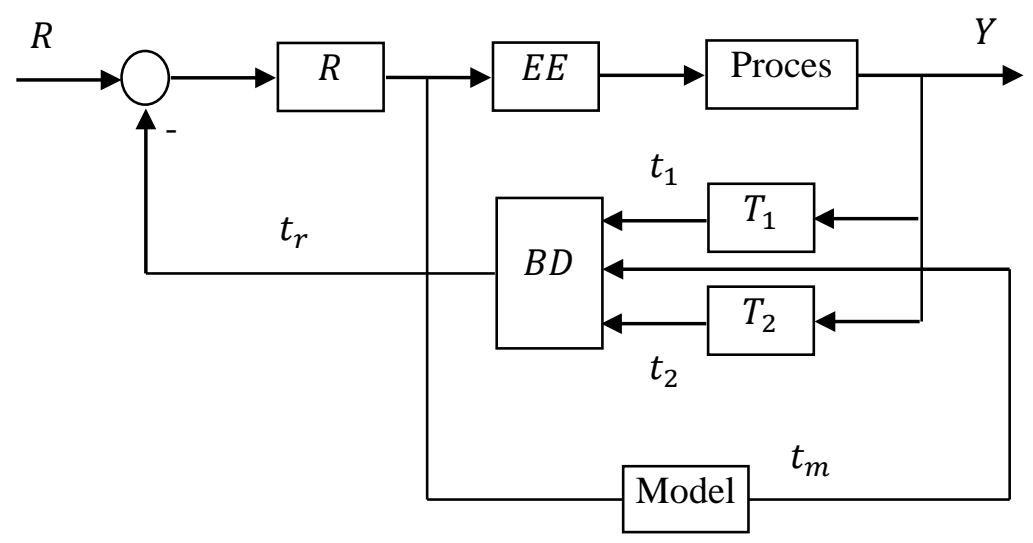

Figure 3 Using a mathematical model for validation transducer

$t_{r}$ signal has the expression:

$t_{r}=k_{1} t_{1}+k_{2} t_{2}$

(3)

Where $\mathrm{k}_{1}$ and $k_{2}$ are coefficients which give a contribution to $\mathrm{T}_{1}$ and $T_{2}$ transducers.

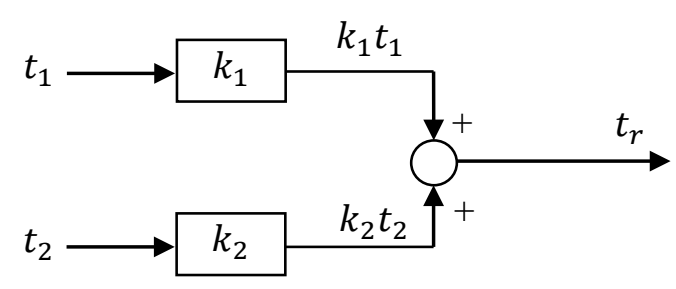

Figure 4 The $t_{r}$ - signal block schema

For normal operation:

$$
t_{1}=t_{2}
$$

and the relation (3) becomes: 


$$
t_{r}=\left(k_{1}+k_{2}\right) t_{1}
$$

It is clear that in this case, the size of reaction must be equal to the signals from transducers, ie:

$$
t_{r}=t_{1}=t_{2}
$$

The coefficients $k_{1}$ and $k_{2}$ are satisfying the condition:

$$
k_{1}+k_{2}=1
$$

Respectively

$$
t_{r}=k_{1} t_{1}+\left(1-k_{1}\right) t_{2}
$$

$k_{1} \in[0,1]$ is a coefficient following a convention:

- $k_{1}=1$ - is where the transducer $T_{2}$ is damaged, and the contribution of $t_{1}$ on the feedback signal $t_{r}$ is $100 \%$;

$-k_{1}=0,5$ - where both the transducer is operating correctly

- $k_{1}=0$ - is if one is damaged $T$ transducer and the contribution of $t_{2}$ signal on the feedback signal $t_{r}$ becomes $100 \%$

To calculate the coefficients $k_{1}$ and $k_{2}$ we introduce a third term $t_{m}$

$$
\begin{aligned}
& k_{1}=\frac{e^{-k_{0}\left|t_{m}-t_{1}\right|}}{e^{-k_{0}\left|t_{m}-t_{1}\right|}+e^{-k_{0}\left|t_{m}-t_{2}\right|}} \\
& k_{2}=\frac{e^{-k_{0}\left|t_{m}-t_{2}\right|}}{e^{-k_{0}\left|t_{m}-t_{1}\right|}+e^{-k_{0}\left|t_{m}-t_{2}\right|}}
\end{aligned}
$$

where $k_{0}$ is a constant whose value can be set depending on the desired changes for the two coefficients. Block diagram is shown in the following figure:

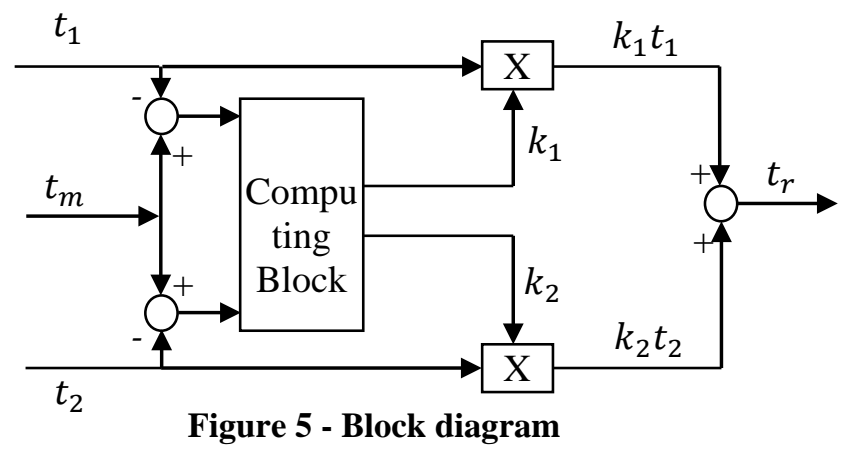

\section{Experiments with Quanser SRV-02 System}

Another option is proposed -derived from the block schema presented in Figure 4 and this is used in the practical experiments.

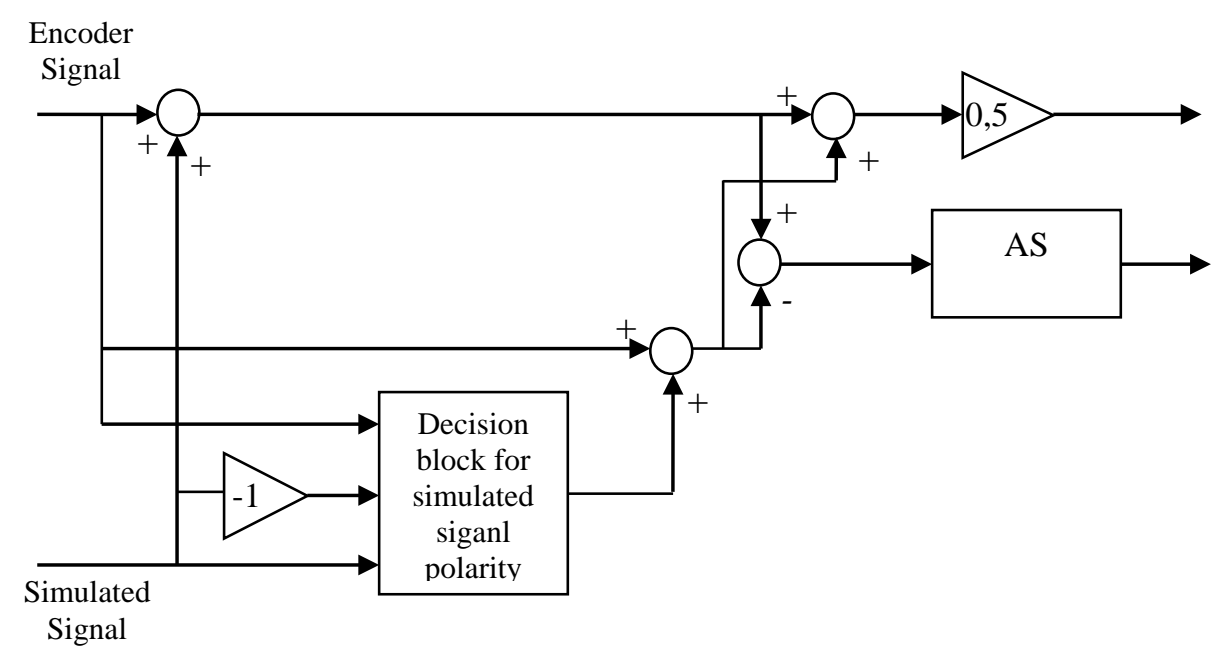




\section{Figure 6}

When the sensor signal is zero, the signal will be simulated with a minus sign.

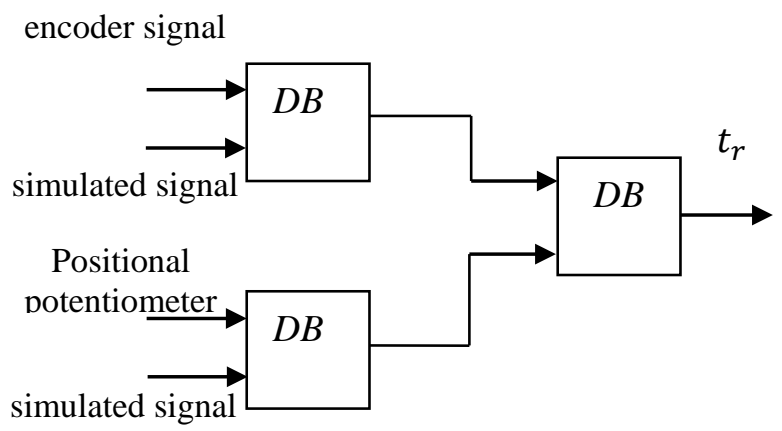

Figure 7 Decision block schema for the real signals

Rotflex system (the Rotary Flexible Joint) with actuator Quanser SRV-02 is shown in figure 8.

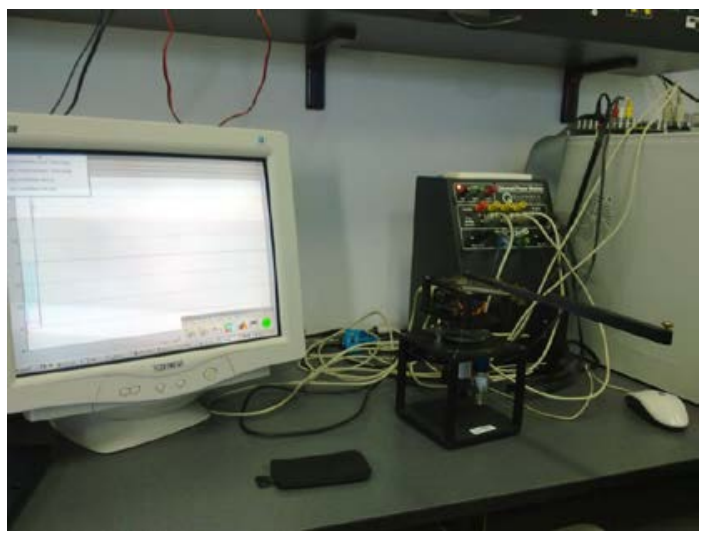

Figure 8 Quanser SRV-02 with the Rotflex system

This system contains an actuator configuration SRV-02 in "high gear" and a joint Rotflex. Rotflex joint contains an encoder whose output shaft is attached a link. It also contains two springs attached link leading to a rotational stiffness around the joint (Quanser Consulting Inc.). [7]

The rotary flexible joint consists of a rotary sensor mounted in a solid aluminum frame and is designed to mount to a Quanser rotary servo plant. The sensor shaft is aligned with the motor shaft. One end of a rigid link is mounted to the sensor shaft. The link rotation is counteracted by two extension springs anchored to the solid frame resulting in an instrumented flexible joint. The spring anchor points are adjustable to three locations to obtain various stiffness constants. Three types of springs are supplied with the system resulting in a total of 9 possible stiffness values. The link is also adjustable in length thus allowing for variations in inertia.

This system is similar in nature to the control problems encountered in large geared robot joints where flexibility is exhibited in the gearbox. A rigid beam is mounted on a flexible joint that rotates via a DC motor. The joint deflection is measured using a sensor. The rotary flexible joint is an ideal experiment intended to model a flexible joint on a robot or spacecraft. This experiment is also useful in the study of vibration analysis and resonance. The simulations were done using MATLAB -Simulink and Wincon plugin. [8]

It is taken into consideration as an iphotesis -that a fault may appear $-\theta$ angle is erroneous - the signal given by the sensors from the real system is faulty. 


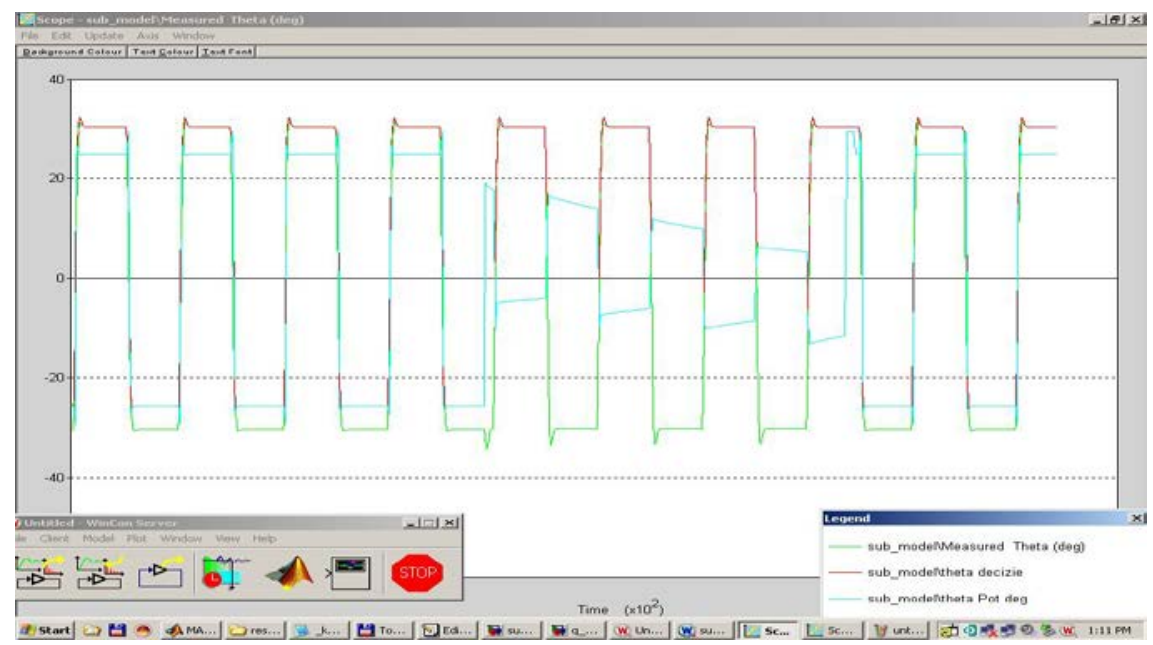

Figure 9 The real signal become faulty and the system is continuing its functioning with the signal from the decision blockwhich gives automatically the simulated signal

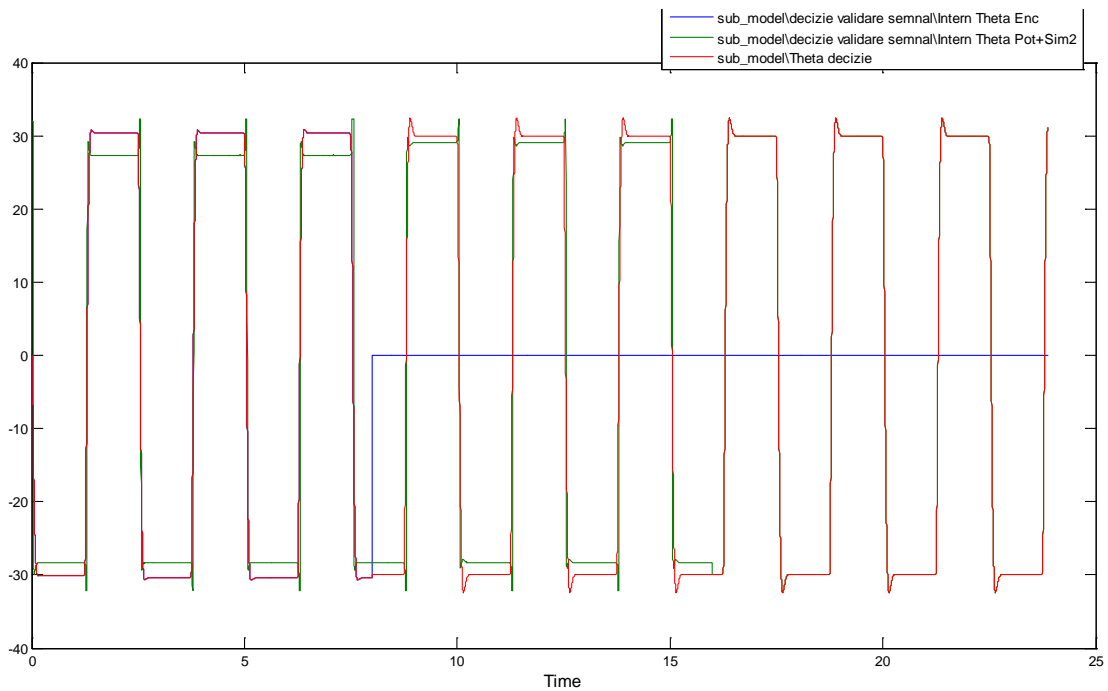

Figure 9 The $\boldsymbol{\theta}$ angle measured by the encoder and also by the positional potentiometer.

From the figure 9 -it can be observed that the fault appears in the 8th second in the signal received from the digital encoder and in the 16th second from the potentiometer signal. The system is controlled only based on the simulated signal.

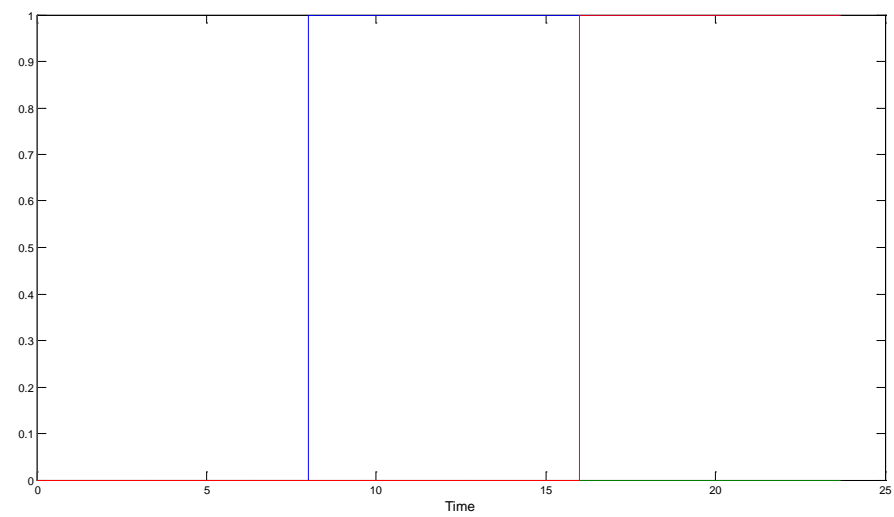

Figure 10 Alarm signal for $\theta$ angle 
The output from the AS (alarm system) block is represented by the blue signal. In the 8th second the signal is changed form 0 to 1 -this means the system is detecting a fault. The red signal represents the corect functioning of the positional potentiometer until the 16th second when the fault appears and the alrm signal comutes to „1”. The green signal reperesnts the simulated signal which continues the process control in the the absence of an unfaulty signal from the sensors of the real system.

\section{Conclusions}

The conclusion that derives from this paper is that both delays can be smaller or greater than the sampling rate $\mathrm{T}$. In the first case, the process can be controlled in optimal conditions. In the second case, major process discontinuity issues can occur which may lead to unsatisfactory evolutions or stability loss.

Following extensive laboratory experiments, the following steps are recommended:

- the evaluation of the dynamic characteristics of the controlled process including actuators and sensors

- sampling rate choice in agreement with the stability and performance criteria

- tests performed on the network used in the control process; medium delays and probability of error are observed

If these delays are much smaller than the necessary sampling rate, the process can be controlled by introducing a delay block in the fixed part of the system.

If the delays become comparable in size with the sampling rate, then the use of a Smith predictor is recommended.

If the network induces large delays and the process has small time constants, remote control is possible only through a hierarchical structure.

\section{Acknowledgment}

This work was supported by the strategic grant POSDRU/159/1.5/S/133255, Project ID 133255 (2014), co-financed by the European Social Fund within the Sectorial Operational Program Human Resources Development 2007 - 2013.

\section{References}

1. Roman M., Bobasu E., Sendrescu D. (2007), Nonlinear Modelling of a Quanser Flexible Link, International Symposium on System Theory , SINTES 13, vol. 1, pp. 169-174

2. Truxal J.G.,Shooman M.J., Blesser W.R. , Clark J.W. (1967), Remote Control, in the Handbook of Telemetry and Remote Control, Mc-Graw Hill, New-York, pp 1-169

3. Iancu E., Vînătoru M., Detecţia şi localizarea defectelor în sisteme dinamice, Edit. Sitech, Craiova, 1999

4. Iancu E., Vînătoru M., Metode analitice pentru detecţia şi localizarea defectelor. Studii de caz, Editura Universitaria, Craiova, ISBN 973-8043-407-6, 2003

5. ***IEEE Standard Dictionary of Electrical and Electronic Terms, the Institute of Electrical and Electronic Engineers, Inc., New York

6. *** Matlab User Guide

7. ***Quanser Consulting Inc., SRV-02 series, Rotary Experiment \#1, Position Control

8. *** Quanser Consulting Inc., SRV-02 series, User Manual, WinCon 5.0 User Manual 